\title{
Mechanism of resistin-induced enhancement of proliferation and migration of ovarian cancer cells
}

\author{
Li Pang, Xian-li Li
}

Department of Obstetrics and Gynecology, Shengjing Hospital of China Medical University, Shenyang, Liaoning, China

Submitted: 15 June 2020

Accepted: 4 October 2020

Arch Med Sci

DOI: https://doi.org/10.5114/aoms.2020.100652

Copyright @ 2020 Termedia \& Banach

\section{Abstract}

Introduction: Resistin, a novel hormone secreted by human adipocytes and mononuclear cells, is associated with obesity, insulin resistance, and inflammation. Recent studies showed that resistin plays a key role in ovarian cancer cells. In this study, we investigated the potential of resistin to regulate the proliferation and migration of ovarian cancer cells.

Material and methods: A series of in vitro functional experiments were carried out to elucidate the role of resistin in ovarian cancer progression and the molecular mechanisms underlying its role.

Results: Resistin enhanced the proliferation of human ovarian epithelial carcinoma cells (HO-8910) in a time- and dose-dependent manner (30-100 ng/ml). Furthermore, HO-8910 cells cultured in adipocyte-conditioned medium showed dramatically increased rates of proliferation. Resistin knockout during adipocyte culture attenuated the proliferation of HO-8910 cells treated with adipocyte-conditioned media, indicating that resistin may promote HO-8910 cell proliferation via the mechanistic target of a rapamycin (mTOR)mediated signaling pathway. Resistin $(30-100 \mathrm{ng} / \mathrm{ml}$ ) also enhanced woundhealing rates in a time- and concentration-dependent manner. Co-culturing HO-8910 cells with adipocytes also increased the wound-healing rates. Resistin expression was inhibited by miR-124-1 transcriptional activity, and resistin-mediated HO-8910 cell migration was also regulated by miR-124-1. Furthermore, we also confirmed the role of resistin in promoting tumor growth in vivo.

Conclusions: These findings suggest that resistin may serve as an effective therapeutic target for ovarian epithelial carcinoma, especially in patients who are obese.

Key words: obesity, adipocyte, mTOR, miR-124.

\section{Introduction}

Ovarian cancer is the second most common gynecologic cancer worldwide. As the ovaries are located in the pelvic cavity and the peritoneum, early-stage ovarian cancer patients are generally asymptomatic. In approximately $70 \%$ of patients, ovarian cancer causes pathological changes beyond the ovaries. The autocrine secretion/self-secretion hypothesis proposes that the activation of oncogenes, such as that in tumor cells, can enable the production of certain growth factors within these tumor cells; this, in turn, promotes tumor proliferation and migration [1]. To develop therapeutic targets, we investigated the mecha-

\author{
Corresponding author: \\ Li Pang \\ Department \\ of Obstetrics and \\ Gynecology \\ Shengjing Hospital \\ of China Medical \\ University \\ Shenyang 110004 \\ Liaoning, China \\ Phone: +24 18940252992 \\ E-mail: \\ doctorpang16@163.com
}


nisms involved in the proliferation and metastasis of ovarian cancer. Resistin is a relatively new cytokine that was discovered in 2001 and plays a role in insulin resistance [2]. High levels of resistin are associated with the development of breast, gastric, colorectal, and esophageal cancers [3-6]. Increased resistin levels are associated with endometrial carcinoma, polycystic ovary syndrome, and uterine cancer [7]. Our preliminary studies indicated that increased resistin expression contributes to the proliferation and migration of ovarian cancer cells; however, this has not been confirmed in any other reported literature in China or abroad. In our present study, we investigated the role of resistin in the proliferation and migration of ovarian cancer cells. Determining the mechanisms underlying resistin actions will promote the development of targeted therapeutics for patients with ovarian cancer.

\section{Material and methods}

\section{Materials}

The HO-8910 human ovarian epithelial carcinoma cell line was purchased from the American Type Culture Collection (ATCC, Manassas, VA, USA). Roswell Park Memorial Institute (RPMI) 1640 medium was purchased from HyClone Co. (Logan, UT, USA). Recombinant resistin was purchased from Phoenix Pharmaceuticals (Burlingame, CA, USA). siRNA (small interfering RNA) was synthesized by Invitrogen using a block-it RNAi design. The JetPEI reagent was purchased from Polyplus-transfection SA (Illkirch, France). All other chemicals and drugs were purchased from Sigma Chemical (St. Louis, MO, USA). Female BALB/C nude mice (4 weeks of age) were obtained from Beijing Huafukang Bioscience Co. Inc (Beijing, China).

\section{Cell culture}

HO-8910 cells were cultured in RPMI 1640 containing 10\% fetal bovine serum (FBS) and penicillin/streptomycin $(100 \mathrm{U} / \mathrm{ml})$ in a humidified $37^{\circ} \mathrm{C}$ incubator. When confluent, cells were treated with resistin $(30-100 \mathrm{ng} / \mathrm{ml})$ for 6 to $48 \mathrm{~h}$. For co-cultured experiments, HO-8910 cells were cocultured with adipocytes and then treated with resistin at $100 \mathrm{ng} / \mathrm{ml}$ for $12 \mathrm{~h}$.

\section{MTT assay}

Briefly, HO-8910 cells were seeded at a density of $1 \times 10^{4}$ cells $/ \mu$ in a 96 -well plate containing DMEM (Dulbecco's modified Eagle medium) and incubated with varying concentrations $(0,10,30$, $100 \mathrm{ng} / \mathrm{ml}$ ) of resistin for 12,24 , or $48 \mathrm{~h}$ at $37^{\circ} \mathrm{C}$. Then, $100 \mu \mathrm{l}$ aliquots containing MTT (3-(4,5-dimethylthiazol-2-yl)-2,5-diphenyl tetrazolium bro- mide; $5 \mathrm{mg} / \mathrm{ml}$ in complete DMEM) were added, and cells were incubated for an additional $5 \mathrm{~h}$. MTT-containing medium was removed and $150 \mu$ of dimethyl sulfoxide (DMSO) was added to dissolve the formazan crystals formed by live cells. Absorbance was measured at $570 \mathrm{~nm}$, and cell viability (\%) was calculated according to the following equation.

\section{Isolation and primary culture of rat adipocytes}

The experimental setup and all animal handling procedures were conducted in accordance with the Animal Handling Guidelines of Shengjing Hospital of China Medical University. Thirty male Wistar rats (Shengjing Hospital Laboratories, Shenyang, China), 6 weeks old, weighing 160-180 g, were housed in two-rat cages with standard bedding material at $21-22^{\circ} \mathrm{C}$ and $50-60 \%$ relative humidity with a 12-h light/dark cycle. Standard rat chow and water were provided ad libitum. After 2 weeks of acclimatization, the rats were sacrificed under isoflurane anesthesia via exsanguination from the exposed aorta using dry heparinized syringes and then rapidly decapitated. Following this, the fat pads were minced into $1 \mathrm{~mm}^{3}$ fragments and transferred to $15 \mathrm{ml}$ centrifuge tubes containing $100 \mu \mathrm{l}$ of collagenase I working solution. The tissues were then incubated in a $37^{\circ} \mathrm{C}$ water bath and agitated at $120 \mathrm{rpm}$ for $50 \mathrm{~min}$. After digestion, the suspensions were filtered through a $250 \mu \mathrm{m}$ mesh filter into tubes containing $5 \mathrm{ml}$ of preheated DMEM per tube. The filtered crude suspension of isolated cells was allowed to stand for $5 \mathrm{~min}$ in stoppered polypropylene syringes at $22-24^{\circ} \mathrm{C}$. The adipocytes floated to form a defined upper layer. Next, the lower aqueous fraction was slowly drained off, and syringes were recapped to retain the adipocytes. The adipocytes were washed three times using this technique. The final supernatant fraction contained intact adipocytes and a free fat layer resulting from broken adipocytes. Working adipocyte samples were acquired from the central layer of adipocytes, ensuring that the free fat layer was excluded.

\section{Wound-healing assay}

HO-8910 cells were seeded onto 24-well plates (at a density of $5 \times 10^{4}$ cells/well) containing RPMI 1640 medium supplemented with $10 \%$ FBS. The cells were incubated for $24 \mathrm{~h}$ until they reached $100 \%$ confluence or had overgrown. A sterile $10 \mu \mathrm{l}$ pipette tip was used to make a scratch (wound) in the center of the wells, after which the wells were washed with phosphate-buffered saline (PBS). Following this, media containing different concentrations of resistin $(0,10,30$, and $100 \mathrm{ng} / \mathrm{ml})$ 
were added to the wells. The culture media were aspirated at 2, 4, 6, and $12 \mathrm{~h}$, washed three times with PBS, and the healing of the wounded area was observed under a microscope. The healed area was evaluated using ImageJ software, and the rate of healing was calculated using the formula: $(\%)=d 1-d 2 / D 1 \times 100 \%$, where $D 1$ represents the wounded area at $0 \mathrm{~h}$ after the scratch, and $D 2$ represents the wounded area after $12 \mathrm{~h}$ of healing. The experiment was repeated three times.

\section{Synthesis and transfection of resistin SiRNA}

Resistin siRNA (Sense: 5'-GGACGUCCGUGAGGAUACAAUGUGU-3'; Antisense: 5'-ACACAUUGUAUCCUCACGGACGUCC-3'; each strand with a 3' dTdT overhang) and negative control siRNA were purchased from Invitrogen Life Technologies. siRNA transfection was performed using Lipofectamine RNAiMAX and Opti-MEM (both from Invitrogen Life Technologies) according to the manufacturer's instructions. On day 2, HO-8910 cells were incubated with $50 \mathrm{nM}$ of each siRNA in a maturation medium and cultured until day 5 .

\section{Plasmid construction and transient transfection assays}

Next, we constructed the fusion gene consisting of human miR-124 promoter and luciferase reporter. First, we purified a series of fragments (positions -1754 to +28 ) of human genomic DNA from peripheral blood, inserted them into pGL3 Basic Luciferase Reporter Vector (Promega, Madison, WI, USA), and designated them as p-miR124. $\mathrm{HO}-8910$ cells were plated in 24 -well plates, transfected with various plasmids via a jetPEl reagent, and incubated for $24 \mathrm{~h}$. Thereafter, the cells were harvested and homogenized. DNA transfection was performed with $0.5 \mu \mathrm{g}$ of fusion reporter genes and $10 \mathrm{ng}$ of the internal reference gene $p R L-T K$ (thymidine kinase promoter - Renilla luciferase reporter; Promega). Luciferase activity was detected using the Dual Luciferase Reporter Assay System (Promega).

\section{Tumor xenograft implantation in nude mice}

Female BALB/c nude mice (4 weeks old) were housed under specific pathogen-free (SPF) conditions. They were randomly divided into three groups (five mice in each group): control group, shRNA-NC group, and sh-resistin group, all of which were injected subcutaneously with $3 \times 10^{6}$ HO-8910 cells. After tumor formation, the width of the tumors in the experimental mice was measured with a pair of Vernier calipers every 3 days. 21 days after implantation, the mice were euthanized by cervical dislocation, the tumors were completely separated and the fat tissues on the tumor surface were removed. The tumors were then photographed and weighed. The tumor volume $(V)$ was calculated using the following formula: $V=\left(\right.$ length $\times$ width $\left.^{2}\right) / 2$. All experiments were carried out in accordance with the Shengjing Hospital and national guidelines and regulations.

\section{Statistical analysis}

All values are presented as the mean \pm standard deviation (SD), and are derived from at least three independent experiments, each performed in triplicate. Data were analyzed using one- or two-way analysis of variance (ANOVA) or Student's t-test (GraphPad Prism, San Diego, CA, USA), depending on the number of groups and variances. Differences were considered statistically significant when the probability was less than $0.05(p<0.05)$.

\section{Results}

\section{Effects of resistin expression on proliferation}

The results of the MTT assay showed that 30-100 $\mathrm{ng} / \mathrm{mL}$ resistin promoted the proliferation of HO-8910 cells in a time- and dose-dependent manner over the course of 24 to $48 \mathrm{~h}$, with proliferation increasing significantly at $48 \mathrm{~h}$ (Figure $1 \mathrm{~A}$ ) $\left({ }^{*} p<0.05,{ }^{* *} p<0.01\right)$.

\section{Interference of siRNA in resistin expression}

Inhibition of resistin expression by SiRNA was demonstrated in rat adipocytes. Real-time quantitative PCR and western blotting analyses showed that resistin levels in adipocytes' mRNA were reduced to $38 \%$ (Figure $1 \mathrm{~B}$ ) and the protein levels were reduced to 32\% (Figure $1 \mathrm{C}$ ).

\section{Effects of differently conditioned media on $\mathrm{HO}-8910$ cell proliferation}

HO-8910 cells were incubated with cultured primary adipose cells, cos-7 cells (used as negative controls), or with harvested adipocyte-conditioned media. The MTT assay results showed that differently conditioned media can enhance the proliferation rate of $\mathrm{HO}-8910$ cells at a given dose within $24 \mathrm{~h}$, compared to cos-7 cell-conditioned medium, which can augment proliferation rates by $48 \mathrm{~h}$ (Figure $1 \mathrm{D} ;{ }^{*} p<0.05,{ }^{* *} p<0.01$ ). Specific knockdown of resistin during adipocyte culture significantly reduced the proliferation-inducing ability of adipocyte-conditioned media. Our results show that adipocyte-conditioned media could significantly promote the proliferation rate of HO-8910 cells, indicating that adipocytokines secreted by adipose tissue may promote the proliferation of HO-8910 cells, particularly in obese patients. 
A

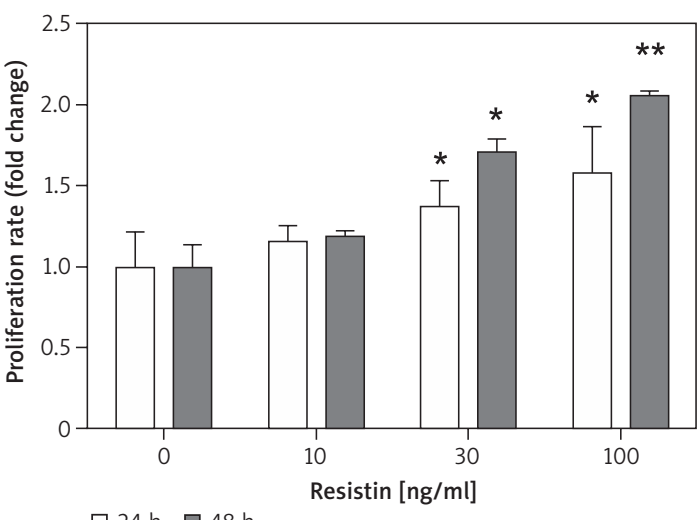

C

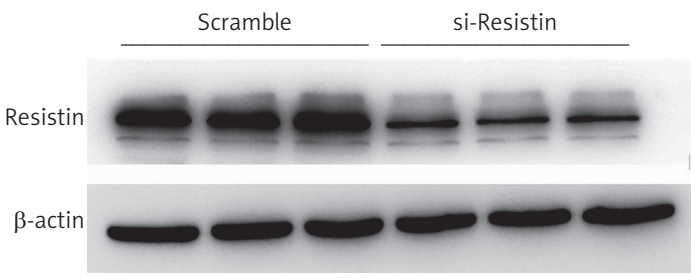

B

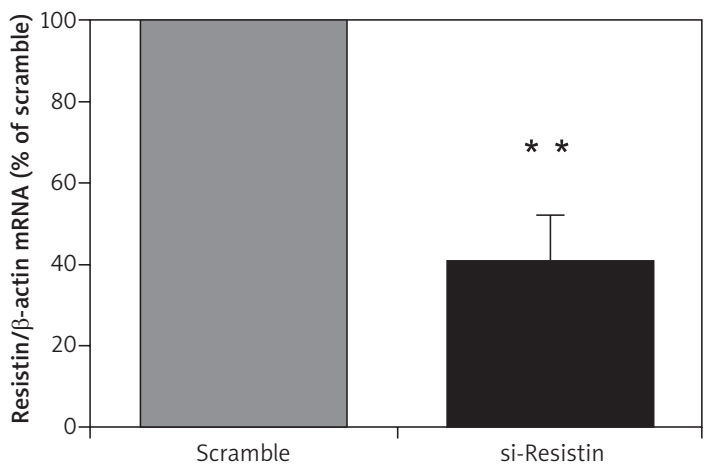

D

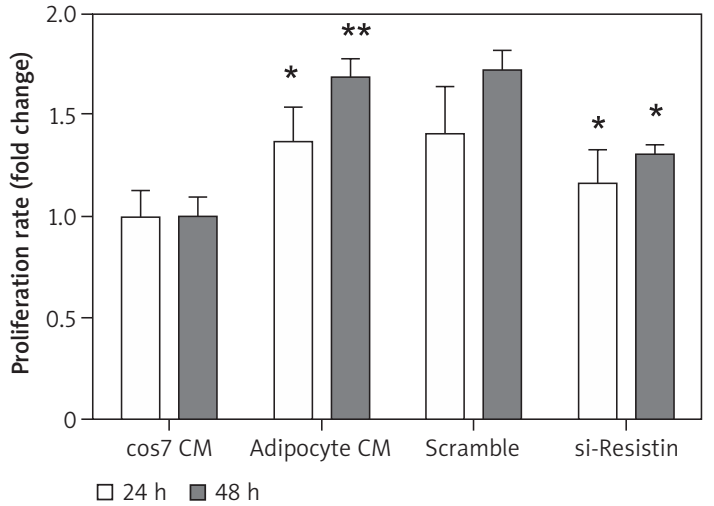

Figure 1. Effect of resistin on the proliferation of ovarian epithelial carcinoma HO-8910 cells. A - Time course of effects of treatment with 30-100 $\mathrm{ng} / \mathrm{ml}$ resistin on the proliferation rate of HO-8910 cells. Dose-response effect of resistin on the proliferation or HO-8910 cells at $48 \mathrm{~h}$ after treatment. B - Small interfering RNA (siRNA) interferes with resistin expression at the mRNA and protein levels. As shown by real-time quantitative PCR, mRNA expression of resistin was decreased to $38 \%$ by resistin knockdown in adipose cells. $\mathbf{C}-$ Western blotting showed that the protein expression of resistin was reduced to $32 \%$ by the knockdown of resistin in adipose cells. D - SiRNA interferes with adipocyte expression of resistin and decreases the proliferative effects of adipocyte-conditioned media. Cos-7 cells were used as negative controls. Adipocyte-conditioned media significantly promoted the proliferation of HO-8910 cells. Knockdown of resistin expression during adipocyte culture attenuated proliferation-inducing effects of adipocyte-conditioned media

\section{Effect of resistin via mTOR signaling pathway}

Although the receptor of resistin has not been identified, mTOR (mechanistic target of rapamycin) is associated with multiple cell-surface receptor types and is implicated in cellular proliferation. To investigate whether mTOR activation was involved in the resistin-mediated response, we used western blotting to detect the phosphorylation of mTOR and its downstream target protein $\mathrm{S} 6$ in $\mathrm{HO}$ 8910 cells. Stimulation with resistin significantly elevated the phosphorylation levels of MTOR and S6 (Figure $2 \mathrm{~A}$ ). Rapamycin is a macrolide immunosuppressant that inhibits mTOR protein kinase. HO-8910 cells were treated with rapamycin $(10,20$, and $30 \mathrm{nM}$ ) and incubated for $24 \mathrm{~h}$. The MTT assay revealed that rapamycin can significantly inhibit HO-8910 cell proliferation rates (control group:
${ }^{*} p<0.05$; resistin-treated cells group: ${ }^{*} p<0.01$ ) (Figure 2 B). Thus, resistin may promote $\mathrm{HO}-8910$ cell proliferation via the mTOR signaling pathway.

\section{Effect of resistin on $\mathrm{HO}-8910$ cell migration}

The wound-healing assay results showed that resistin promoted HO-8910 cell migration. Resistin $(100 \mathrm{ng} / \mathrm{ml})$ induced cell healing in a time- and dose-dependent manner. As early as $6 \mathrm{~h}$ after wound induction, the healing rate increased to $42.59 \pm 3.94 \%$ and remained elevated for up to 12 h $(66.61 \pm 4.69 \%)$ (Figures 3 A, B). Treatment with resistin (30-100 $\mathrm{ng} / \mathrm{ml}$ ) for $12 \mathrm{~h}$ also caused a concentration-dependent increase in the woundhealing rate $(43.31 \pm 1.28 \%, 53.65 \pm 6.01 \%, 66.61$ $\pm 4.69 \%)$ (Figures 3 C, D) $\left({ }^{*} p<0.05,{ }^{* *} p<0.05\right)$.

The wound-healing assay suggested that the migration of HO-8910 cells co-cultured with resis- 
A

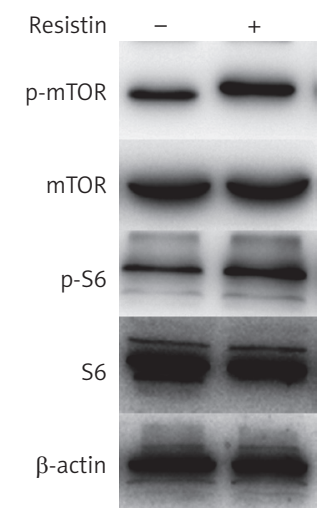

B

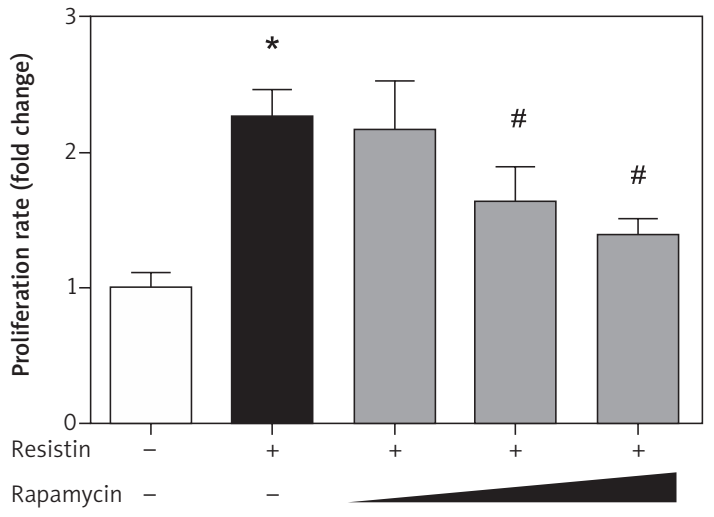

Figure 2. Involvement of mTOR (mechanistic target of rapamycin) signaling pathway in resistin-mediated induction of HO-8910 proliferation. A - Stimulation with resistin significantly elevated the phosphorylation of mTOR and S6 in HO-8910 cells. B - The mTOR inhibitor rapamycin significantly inhibited proliferation of HO-8910 cells that were pretreated with resistin

tin-pretreated adipocytes was enhanced, exhibiting a wound-healing rate of $44.88 \pm 3.83 \%$. Resistin knockdown in the co-culture system significantly reduced the wound-healing rate (30.91 $\pm 3.21 \%$; ${ }^{*} p<0.05$ ) (Figures 3 E, F). HO-8910 cells were then incubated with cultured primary adipocytes, cos7 cells (used as negative controls), or harvested adipocyte-conditioned media. The adipocyte-conditioned media significantly enhanced the healing rate of HO-8910 cells compared to that induced by cos-7 cell-conditioned media (48.39 $\pm 2.88 \%$; $\left.{ }^{*} p<0.05\right)$. Similarly, resistin knockdown in the coculture system significantly reduced the woundhealing rate induced by adipocyte-conditioned media (36.33 $\pm 2.01 \%$; $\left.{ }^{*} p<0.05\right)$ (Figures $3 \mathrm{G}, \mathrm{H}$ ).

\section{Effects of miR-124 on resistin-treated HO-8910 cell migration}

We examined the effect of resistin on miR-124 expression and found that resistin significantly inhibited the expression of miR-124-1, but not that of miR-124-2 and miR-124-3 (Figure 4 A). Next, we cloned the gene sequence of miR-124-1 and subcloned it into the pGL3 Basic Luciferase Reporter Vector. We then measured the relative luciferase activity in the transiently transfected HO-8910 cells. Our results indicated that resistin treatment significantly inhibited the activity of miR-124-1 in HO-8910 cells (Figure 4 B). The exact mechanisms of the effects of miR-124 on migration must be further elucidated and will be addressed in our future studies.

\section{Resistin promotes tumor growth in vivo}

HO-8910 cells with sh-driven resistin knockdown were used to model the in vivo effects of resistin on the growth of tumors in nude mice.
The results showed that tumors in the mice from the sh-resistin group, which were subcutaneously implanted with resistin-deficient HO-8910 cells, formed later, grew slower, and had significantly smaller volumes and weights compared with those in mice from the shRNA-NC and the control groups $\left({ }^{\star} p<0.05\right.$; Figure 5$)$.

\section{Discussion}

This study's results demonstrated that treatment with resistin promoted $\mathrm{HO}-8910$ cell proliferation via the mTOR pathway and migration via the miR-124-1 pathway. Resistin upregulated the proliferation of HO-8910 cells in a time- and concentration-dependent manner. Knockdown of resistin expression via siRNA treatment reduces the proliferation rate of $\mathrm{HO}-8910$ cells. This resistinmediated increase in $\mathrm{HO}-8910$ cell proliferation occurred via the mTOR signaling pathway, possibly through a paracrine/self-secretion effect. Resistin also upregulated the wound-healing rate in $\mathrm{HO}$ 8910 cells in a time- and concentration-dependent manner and reduced the activity of the miR-1241 promoter. To the best of our knowledge, this is the first report demonstrating resistin-mediated signaling pathways involved in the promotion of proliferation and migration of ovarian cancer cells.

Ovarian cancer, one of the five types of malignancy observed in the female reproductive system, is often diagnosed too late for effective treatment. Although the diagnostic rate of ovarian cancer has been improving with advances in surgical techniques, chemotherapy, and targeted therapy, the spread of ovarian cancer to other organs is a key problem that remains to be solved. A study conducted in the United States showed that women with a normal weight experience lower mortality rates than those with obesity [8]. Central or ab- 
A

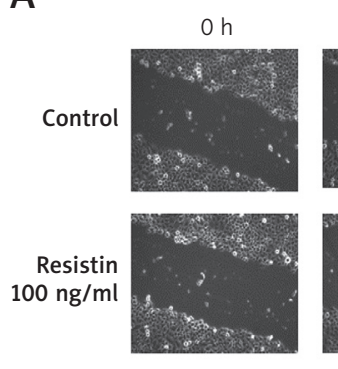

C

Resistin

$\mathrm{Oh}$

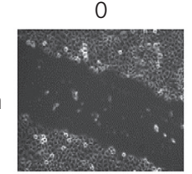

$12 \mathrm{~h}$

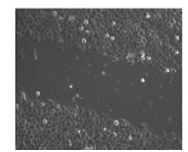

E
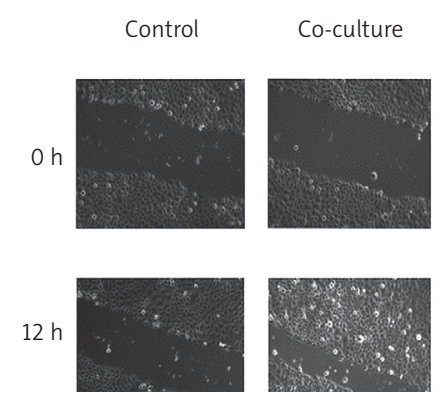

G
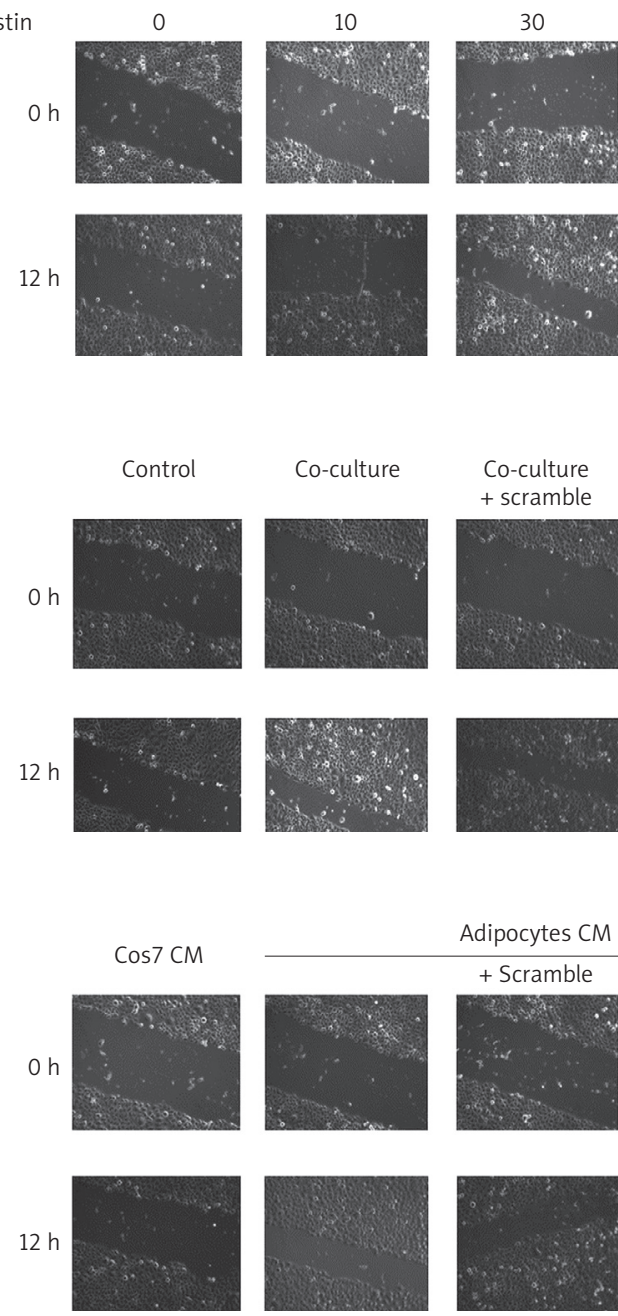

Adipocytes CM

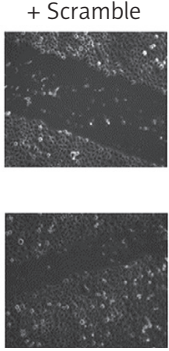

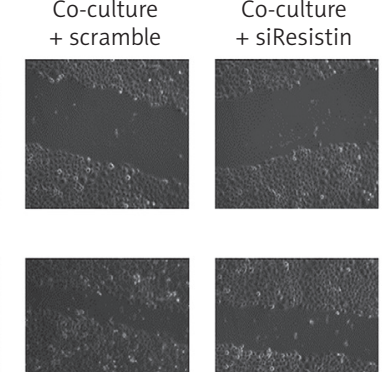

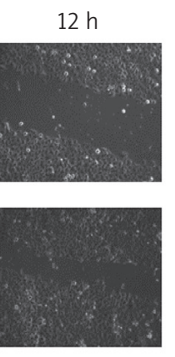

B

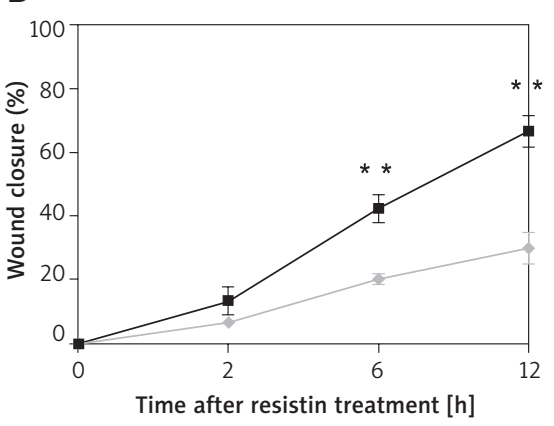

D
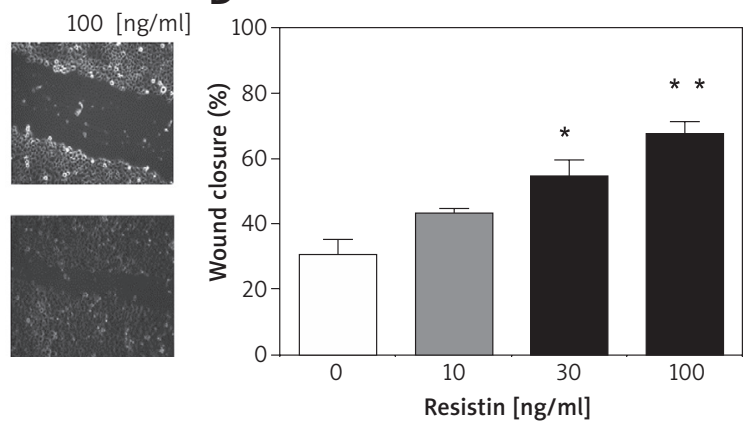

F
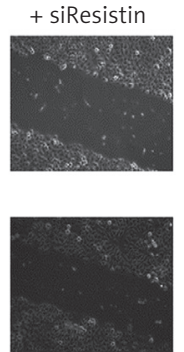

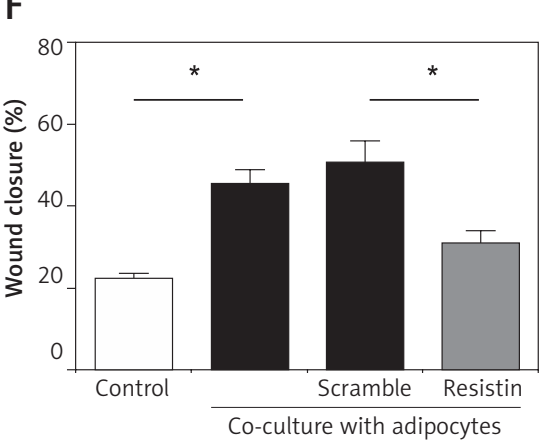

$\mathrm{H}$

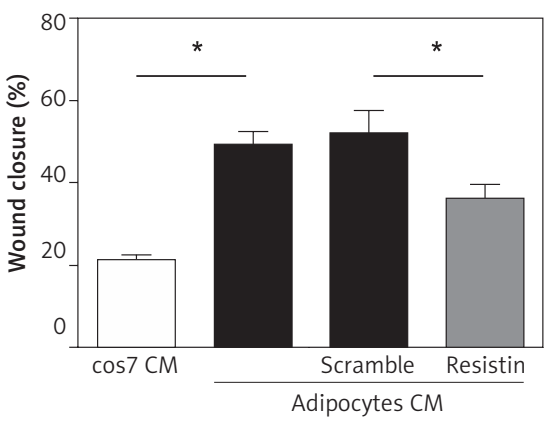

Figure 3. Effect of resistin on HO-8910 cell migration and interference of siRNA in the resistin-mediated effect on co-culture migration. A, B - Time course of the effects of treatment with $100 \mathrm{ng} / \mathrm{ml}$ resistin on the wound-healing rates of HO-8910 cells. C, D - Dose-response effect of $12 \mathrm{~h}$ treatment with resistin on the wound-healing rate of HO-8910 cells. E, F - The wound-healing rate with resistin compared to that with the knockout of resistin in the co-culture system. Co-cultured cells were incubated with $100 \mathrm{ng} / \mathrm{ml}$ resistin for $12 \mathrm{~h}$. G, H-Cos-7 cells were used as negative controls; adipocyte-conditioned media significantly promoted the wound-healing rate of HO-8910 cells. The wound-healing rate was attenuated by the knockout of resistin in a co-culture system 
A

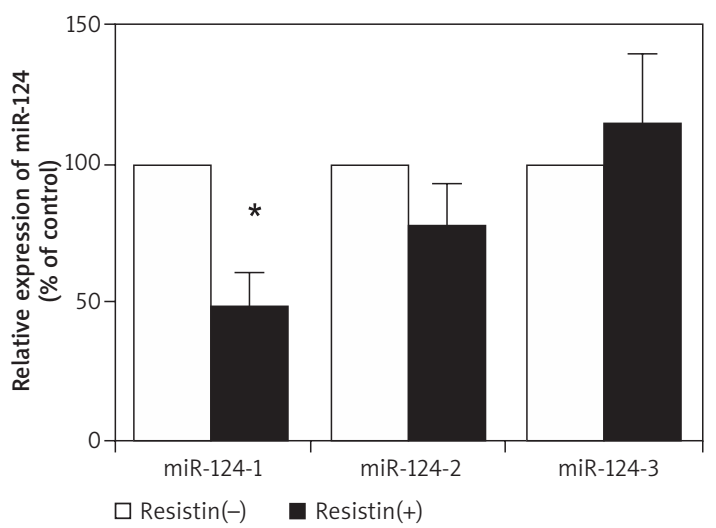

B

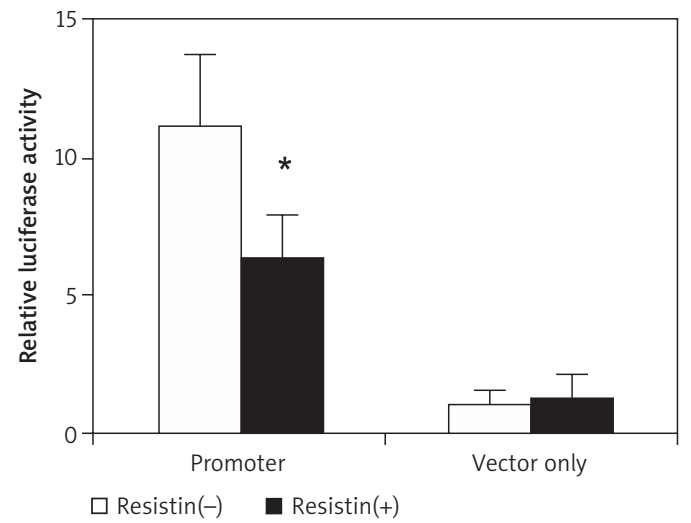

Figure 4. Effect of miR-124 on resistin expression and HO-8910 cell migration. A - The effect of resistin on miR-124 expression. HO-8910 cells were pretreated with or without resistin, then incubated with $100 \mathrm{ng} / \mathrm{ml}$ resistin for $24 \mathrm{~h}$. miR-124-1 expression was attenuated by resistin in HO-8910 cells. MiR-124-2 and miR-124-3 expression was not affected by resistin. B - Effects of miR-124 on resistin promoter activity in HO-8910 cells

A

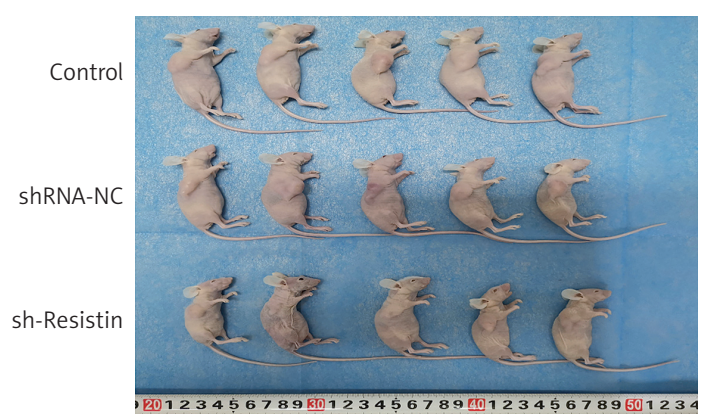

C

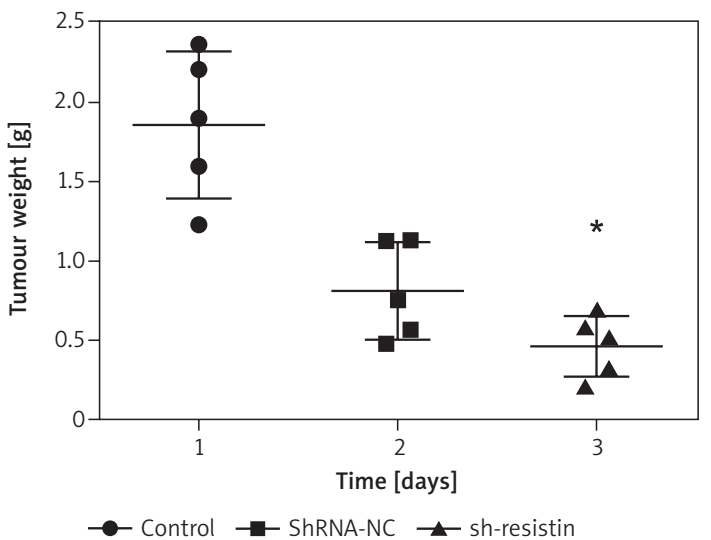

B

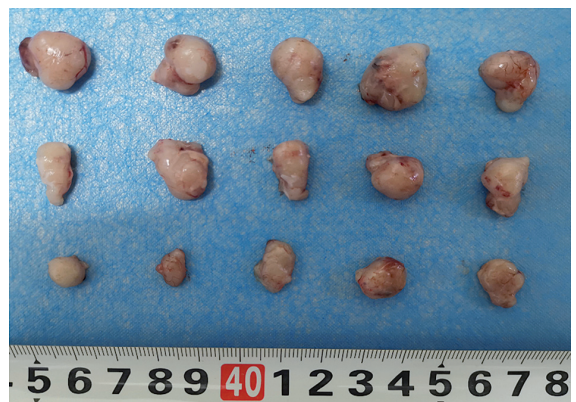

D

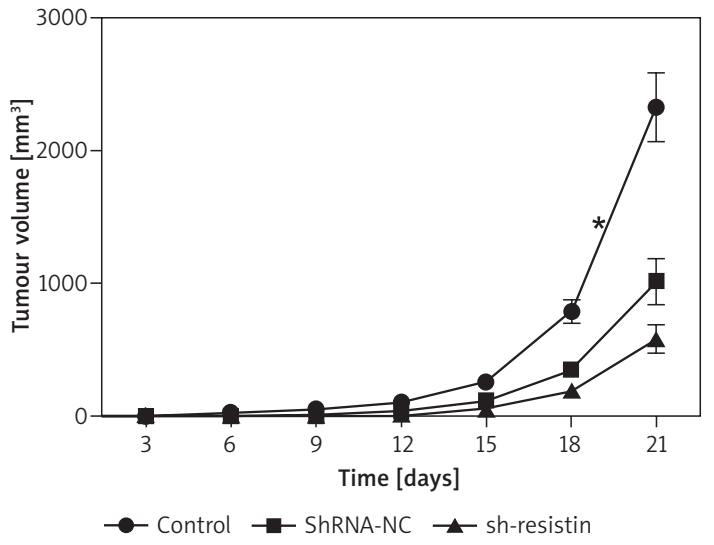

Figure 5. In vivo tumor xenograft study to elucidate the role of resistin. A, B - Nude mice from each group that showed tumor growth following the transplantation of control, shRNA-NC-transfected, and sh-resistin-transfected HO-8910 cells. C, D - Tumor volume was calculated every 3 days after injection, and the tumors were excised and weighed after 21 days $\left({ }^{*} p<0.05\right)$

dominal obesity is gaining popularity as a key risk factor for cardiovascular disease (CVD) and higher mortality rates $[9,10]$. Obese patients show a low survival rate and a high risk of cancer recurrence, regardless of the administration of chemotherapy or other auxiliary therapies. However, the role of obesity in tumor development is unclear. Obesity causes a chronic, low-grade inflammatory state in which high levels of inflammatory adipokines are secreted by adipocytes. Adipocyte factors such as leptin, tumor necrosis factor $\alpha$, interleukin- 6 , and adiponectin are closely associated with tumor development, especially in women with endometrial and breast cancer [11, 12]. Adipocytes can also 
promote tumor progression in ovarian cancer. Nieman et al. reported that retinal adipocytes can promote the proliferation, migration, and invasion of ovarian cancer cells. Adipocytes can also serve as a source of energy for tumors and promote the proliferation of ovarian cancer cells [13]. Adipocytes in the ovarian cancer microenvironment may produce molecules that assist cancer cells in the maintenance of their malignant phenotypes. Furthermore, adipose tissue can secrete hormones or other types of proteins that promote the excessive growth of ovarian cancer cells. Previous studies have shown that obesity impacts ovarian cancer-induced mortality by influencing tumor biology; however, the relationship between ovarian cancer-induced mortality and obesity needs further research.

Resistin is an adipocytokine discovered in 2001 and named for its role in insulin resistance [2]. Current studies on resistin have mainly examined its roles in inflammation and insulin resistance. When insulin levels are constant, resistin can stimulate glucose output. Mice with hereditary and diet-induced obesity demonstrate significantly increased plasma resistin levels. In humans, resistin is not only secreted by adipocytes, but also expressed by peripheral blood monocytes [14]. Epidemiological studies show that plasma resistin levels are positively correlated with BMI. High levels of resistin are associated with the development of breast, gastric, colorectal, and esophageal cancer. High resistin expression is also associated with more malignant clinicopathological statuses and adverse prognoses [15]. Regarding gynecological diseases, studies have focused on the role of resistin in polycystic ovary syndrome. Increased resistin expression is also closely related to the development of choriocarcinoma and endometrial carcinoma. However, few studies have examined the role of resistin in ovarian cancer. Recently, Qiu et al. reported that increased resistin expression increases growth, invasiveness, and cisplatin resistance in ovarian cancer cells. The mechanisms driving this activity include the induction of epithelial-mesenchymal transition (EMT) and stemness, as evidenced by the downregulated expression of the epithelial marker e-cadherin and the upregulated expression of mesenchymal markers vimentin/ZEB1 and stemness markers sox2, oct4, and nanog [16]. Previously, we demonstrated that resistin promotes the angiogenesis of ovarian cancer cells [17]. This study showed that resistin promoted the proliferation of epithelial ovarian HO-8910 cancer cells. Knockdown of resistin in adipocyte cultures significantly decreased the proliferation of HO-8910 cells that were treated with the media used in thOur results indicate that resistin induced acti- vation of the mTOR pathway and phosphorylation of the mTOR downstream target protein $\mathrm{S} 6$ in HO-8910 cells, whereas rapamycin exerted the opposite effect on mTOR activation. This shows that mTOR activation may be important for the proliferation of ovarian cancer cells, and that resistin promotes proliferation of ovarian cancer cells by activating mTOR. Our results suggest that the mTOR signaling pathway may be a therapeutic target for treatment of obese patients with ovarian cancer.

mTOR is a key cellular molecule that determines the energy levels inside and outside the cell, regulates transcription and translation, and mediates cell and tissue responses during survival, growth, proliferation, and differentiation. Whereas mTOR inhibits the activity of its downstream target protein $\mathrm{S6}$, rapamycin inhibits the activity of anabolic S6 ribosomal proteins and increases the transcription of proliferating cell nuclear antigen (PCNA), thereby regulating the cell cycle and inhibiting the proliferation of smooth muscle and endothelial cells and fibroblasts. Rapamycin also inhibits the transcription of proto-oncogene $\mathrm{BCl}-2$, which plays a key role in the cell cycle. Inhibition of the PI3K/ AKT/mTOR signaling pathway is deemed a striking opportunity for the development of novel anticancer agents [18]

Our current study shows that resistin promoted the migration of epithelial ovarian cancer cells (HO-8910) as observed in the wound assay. Cell migration was attenuated by knocking down resistin expression in HO-8910 cells. Conversely, co-culturing ovarian cancer cells and adipocytes significantly promoted the migration of HO-8910 cells. Cell migration was attenuated by knocking down resistin expression in co-cultured cells. These results further showed that resistin plays an important role in the development and progression of ovarian cancer.

Similar to protein-encoding genes, miRNA is involved in important biological functions, and numerous miRNAs play vital roles in tumorigenesis and tumor inhibition. Tumorigenesis-associated miRNAs tend to promote cell proliferation, inhibit apoptosis and activity of immune cells, and control cell cycles. Suppressor miRNAs tend to inhibit cell growth and promote apoptosis. Multiple factors are involved in the migration and invasion of tumor cells. Recent studies have shown that endogenous non-coding small-molecule RNAs (microRNAs) play important roles in regulating gene transcription and protein translation. Among these miRNAs, miR-124 helps inhibit tumor invasiveness [19]. Studies show that miR-124 expression is downregulated in liver and cervical cancer at the cellular and tissue level, which inhibits the growth and infiltration of myoblastoma and oral 
squamous cell carcinoma. miR-124 may act as a potential tumor suppressor and is known to exert important regulatory effects on cell migration during embryonic stem cell differentiation. In our present study, resistin significantly inhibited the expression of miR-124. Furthermore, observations from in vivo tumorigenesis experiments in nude mice also verified that the expression of resistin was positively correlated with the tumorigenic capacity of the cells. Our results suggest that resistin may reverse the inhibitory effects of miR-124, thereby promoting the migration ability of ovarian cancer cells. The validation of this notion, however, requires further studies.

In conclusion, the results of our present study suggest that targeting resistin, mTOR, and miR124 expression may be a beneficial strategy for the treatment of patients with ovarian epithelial carcinoma, particularly in those with concurrent obesity. We found that resistin is involved in the progression of ovarian cancer and in proliferation and migration of ovarian cancer cells. Therefore, the mechanisms involved in resistin-mediated signaling may present new therapeutic targets in halting the development and migration of ovarian cancer. Our study also illustrates a new mechanism that promotes the migratory ability of ovarian cancer cells. Resistin promoted the proliferation of these cells via the mTOR pathway and their migration via regulating miR-124 activity. These results show that mTOR and miR-124 can be used as therapeutic targets in the treatment and prevention of metastasis in patients with ovarian cancer. We focused on the ability of resistin to promote the proliferation and invasion of ovarian cancer, and have only elucidated one of the potential pathways. Further research is required to uncover the other pathways that govern resistinmediated effects in ovarian cancer, so that therapeutic targeting of resistin may be achieved in obese patients with ovarian cancer.

\section{Conflict of interest}

The authors declare no conflict of interest.

\section{References}

1. Billottet C, Janji B, Thiery JP, Jouanneau J. Rapid tumor development and potent vascularization are independent events in carcinoma producing FGF-1 or FGF-2. Oncogene 2002; 21: 8128-39.

2. Steppan CM, Bailey ST, Bhat S, et al. The hormone resistin links obesity to diabetes. Nature 2001; 409: 307-12.

3. Wang CH, Wang PJ, Hsieh YC, et al. Resistin facilitates breast cancer progression via TLR4-mediated induction of mesenchymal phenotypes and stemness properties. Oncogene 2018; 37: 589-600.

4. Jiang R, Zhao C, Wang X, et al. Resistin-like moleculebeta promotes invasion and migration of gastric carcinoma cells. Med Sci Monit 2016; 22: 937-42.
5. Huang WS, Yang JT, Lu CC, et al. Fulvic acid attenuates resistin-induced adhesion of HCT-116 colorectal cancer cells to endothelial cells. Int J Mol Sci 2015; 16: 29370-82.

6. Nakajima TE, Yamada Y, Hamano T, et al. Adipocytokines and squamous cell carcinoma of the esophagus. Cancer Res Clin Oncol 2010; 136: 261-6.

7. Yang HS, Yoon C, Myung SK, Park SM. Effect of obesity on survival of women with epithelial ovarian cancer: a systematic review and meta-analysis of observational studies. Int J Gynecol Cancer 2011; 21: 1525-32.

8. Pavelka JC, Brown RS, Karlan BY, et al. Effect of obesity on survival in epithelial ovarian cancer. Cancer 2006; 107: 1520-4.

9. Yamout R, Ammar W, Tabbara J, Fares S, Assi M, Sibai AM. Waist circumference cutoff points for predicting metabolic abnormalities in Lebanese adults. Arch Med Sci Civil Dis 2019; 4: e64-71.

10. Mariscalco G, D’Errigo P, Biancari F, et al. Early and late outcomes after transcatheter versus surgical aortic valve replacement in obese patients. Arch Med Sci 2020; 16: 796-801.

11. McCourt CK, Mutch DG, Gibb RK, et al. Body mass index: relationship to clinical, pathologic and features of microsatellite instability in endometrial cancer. Gyneco Oncol 2007; 104: 535-9.

12. Harvie M, Hooper L, Howell AH. Central obesity and breast cancer risk: a systematic review. Obes Rev 2003; 4: 157-73.

13. Nieman KM, Kenny HA, Penicka CV, et al. Adipocytes promote ovarian cancer metastasis and provide energy for rapid tumor growth. Nat Med 2011; 17: 1498-503.

14. Lee YC, Chen YJ, Wu CC, Lo S, Hou MF, Yuan SS. Resistin expression in breast cancer tissue as a marker of prognosis and hormone therapy stratification. Gynecol Oncol 2012; 125: 742-50.

15. Patel L, Buckels AC, Kinghorn IJ, et al. Resistin is expressed in human macrophages and directly regulated by PPAR gamma activators. Biochem Biophys Res Comm 2003; 300: 472-6.

16. Qiu L, Zhang GF, Yu L, Wang HY, Jia XJ, Wang TJ. Novel oncogenic and chemoresistance-inducing functions of resistin in ovarian cancer cells require miRNAs-mediated induction of epithelial-to-mesenchymal transition. Sci Rep 2018; 8: 12522.

17. Pang L, Zhang Y, Yu Y, Zhang S. Resistin promotes the expression of vascular endothelial growth factor in ovary carcinoma cells. Int J Mol Sci 2013; 14: 9751-66.

18. Zhao S, Shao L, Wang Y, Meng Q, Yu J. Ketamine exhibits anti-gastric cancer activity via induction of apoptosis and attenuation of PI3K/Akt/mTOR Arch Med Sci 2020; 16: 1140-9.

19. Hatziapostolou M, Polytarchou C, Aggelidou E, et al. An HNF4alpha-miRNA inflammatory feedback circuit regulates hepatocellular oncogenesis. Cell 2011; 147: 1233-47. 